\title{
Experimental Study of the Statistical Properties of Directionally Spread Ocean Waves Measured by Buoys
}

\author{
M. L. MCAlLiSTER AND T. S. VAN DEN BREMER \\ Department of Engineering Science, University of Oxford, Oxford, United Kingdom
}

(Manuscript received 17 September 2019, in final form 10 November 2019)

\begin{abstract}
Wave-following buoys are used to provide measurements of free surface elevation across the oceans. The measurements they produce are widely used to derive wave-averaged parameters such as significant wave height and peak period, alongside wave-by-wave statistics such as crest height distributions. Particularly concerning the measurement of extreme wave crests, these measurements are often perceived to be less accurate. We directly assess this through a side-by-side laboratory comparison of measurements made using Eulerian wave gauges and model wave-following buoys for randomly generated directionally spread irregular waves representative of extreme conditions on deep water. This study builds on the recent work of McAllister and van den Bremer (2019, https://doi.org/10.1175/JPO-D-19-0170.1), in which buoy measurements of steep directionally spread focused waves groups were considered. Our experiments confirm that the motion of a wave-following buoy should not significantly affect the measured wave crest statistics or spectral parameters and that the discrepancies observed for in situ buoy data are most likely a result of filtering. This filtering occurs when accelerations that are measured by the sensors within a buoy are converted to displacements. We present an approximate means of correcting the resulting measured crest height distributions, which is shown to be effective using our experimental data.
\end{abstract}

\section{Introduction}

Wave-following measurement buoys are widely deployed across the oceans, as they provide a cost effective, easy-toinstall alternative to their Eulerian counterparts. As a result of this, they represent an abundant source of data and metocean statistics, outnumbering Eulerian observations by an order of magnitude (Christou and Ewans 2014). However, within the oceanographic community, it is generally perceived that the measurements these devices produce are less accurate particularly when considering the measurement of extreme wave crests (Allender et al. 1989; Seymour and Castel 1998; Forristall 2000; Dysthe et al. 2008; Collins et al. 2014; Christou and Ewans 2014).

One reason for this perception is the apparent linearization of wave crests measured by buoys (Casas-Prat and Holthuijsen 2010), removing so-called bound harmonics that occur at second order in wave steepness. An example of this is given in Fig. 1. This figure presents data measured by a Datawell Waverider buoy located in

Corresponding author: M. L. McAllister, mark.mcallister@ eng.ox.ac.uk the North Pacific Ocean $\left(33^{\circ} 13^{\prime 2} 29^{\prime \prime} \mathrm{N}, 119^{\circ} 52^{\prime} 54^{\prime \prime} \mathrm{W}\right)$ and operated by the Coastal Data Information Programme (CDIP, see appendix A for full details of the dataset used). Figure 1a presents the probability of exceedance of a given crest height $\eta_{c}$ as function of normalized crest height $\eta_{c} / H_{s}$; we define significant wave height $H_{s}$ as $4 \sqrt{m_{0}}$, where $m_{0}$ is the zeroth spectral moment. Individual crest heights measured by the buoy are shown as blue markers; these observations are predicted well by the Rayleigh distribution,

$$
P\left(\eta_{c}>\eta\right)=\exp \left[-8\left(\frac{\eta}{H_{s}}\right)^{2}\right],
$$

as shown by the solid black line. However, for steep waves in the oceans, vertical skewness that is created by second-order bound nonlinearity increases the probability of large wave crests beyond that of a Rayleigh distribution, which describes a linear Gaussian process. The dotted line shows one of the many (e.g., Tayfun 1980; Forristall 2000; Prevosto et al. 2000; SocquetJuglard et al. 2005) available probability distributions that use observed significant wave height $H_{s}$, mean pe$\operatorname{riod} T_{1}=m_{0} / m_{1}$, and water depth $d$ to account for this second-order nonlinearity (Forristall 2000), 


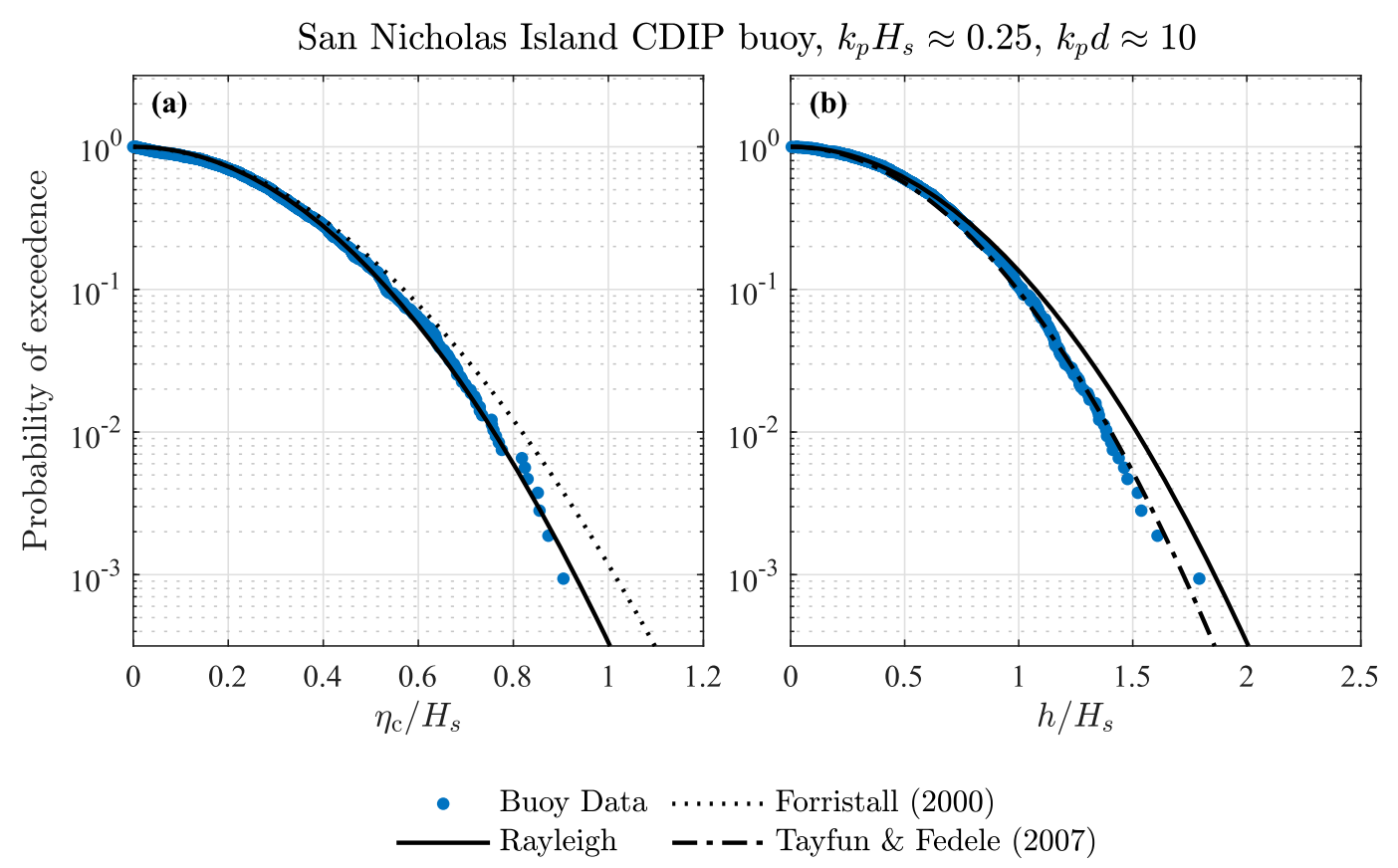

FIG. 1. (a) Crest and (b) wave height exceedance probability for in situ buoy measurements made using a Datawell Waverider buoy moored beside San Nicholas Island in the North Pacific. Blue markers denote individual crest and wave heights measured by the buoy; in (a) the black solid and dotted lines show Rayleigh (1) and Forristall (2) distributions for crest height, respectively, and in (b) the black solid and dashed lines show Rayleigh (8) and bandwidth-corrected Rayleigh [(3); Tayfun and Fedele 2007] distributions for wave height, respectively.

$$
P\left(\eta_{c}>\eta\right)=\exp \left[-\left(\frac{\eta}{\alpha H_{s}}\right)^{\beta}\right],
$$

using the Weibull parameters $\alpha\left(H_{s}, T_{1}, d\right)$ and $\beta\left(H_{s}, T_{1}\right.$, d) (see appendix B). For the conditions in Fig. 1 (and those we will examine experimentally herein), the Forristall distribution gives near identical results to the approximate version of the Tayfun (1980) distribution proposed by Socquet-Juglard et al. (2005). For the buoy data we examine, the crest height follows the linear Rayleigh distribution (1) and is over predicted by the secondorder Forristall distribution (2). Second-order distributions such as (2) have been validated using Eulerian in situ observations, and their accuracy for Eulerian measurements is relatively well established (Forristall 2000; Christou and Ewans 2011). Moreover, for extreme experimental conditions, second-order crest height distributions have even been shown to underpredict crests (Onorato et al. 2009; Latheef and Swan 2013).

Figure $1 \mathrm{~b}$ presents the probability of exceedance of a given wave height $h$ as function of normalized wave height $h / H_{s}$. The blue markers show the zeroupcrossing wave height of each wave measured by the buoy, which is well predicted by the bandwidth-corrected Rayleigh distribution shown by the dash-dotted black line (Boccotti 1981),

$$
P\left(h_{u}>h\right)=\exp \left[-\frac{4}{1+\Psi}\left(\frac{h}{H_{s}}\right)^{2}\right],
$$

where the parameter $\Psi$ accounts for bandwidth and is defined as the value of the envelope of the autocorrelation function of the underlying wave spectrum at $T_{1} / 2$ (Tayfun and Fedele 2007). Unlike wave crests, secondorder bound nonlinearity does not affect wave height, and hence wave height follows a bandwidth-corrected Rayleigh distribution in the absence of higher-order effects (Longuet-Higgins 1952).

Summarizing, the in situ buoy measurements we examine follow the expected wave height distribution [(3); Tayfun and Fedele 2007] but not the expected crest height distribution [(2); Forristall 2000]; this is also observed in Casas-Prat and Holthuijsen (2010) for a much larger dataset (containing $N=10^{6}$ waves). Several studies have suggested that a buoy's mooring or its freedom to move horizontally and avoid large crests (Allender et al. 1989; Krogstad and Barstow 2000) may result in this apparent underestimation of extreme wave crests. However, if either of these two effects were the cause of preferential avoidance of wave crests, one may expect that the measured (crest-to-trough) wave height would also be affected, which we do not observe. In previous work, we have shown the latter of these two effects (the avoidance of 


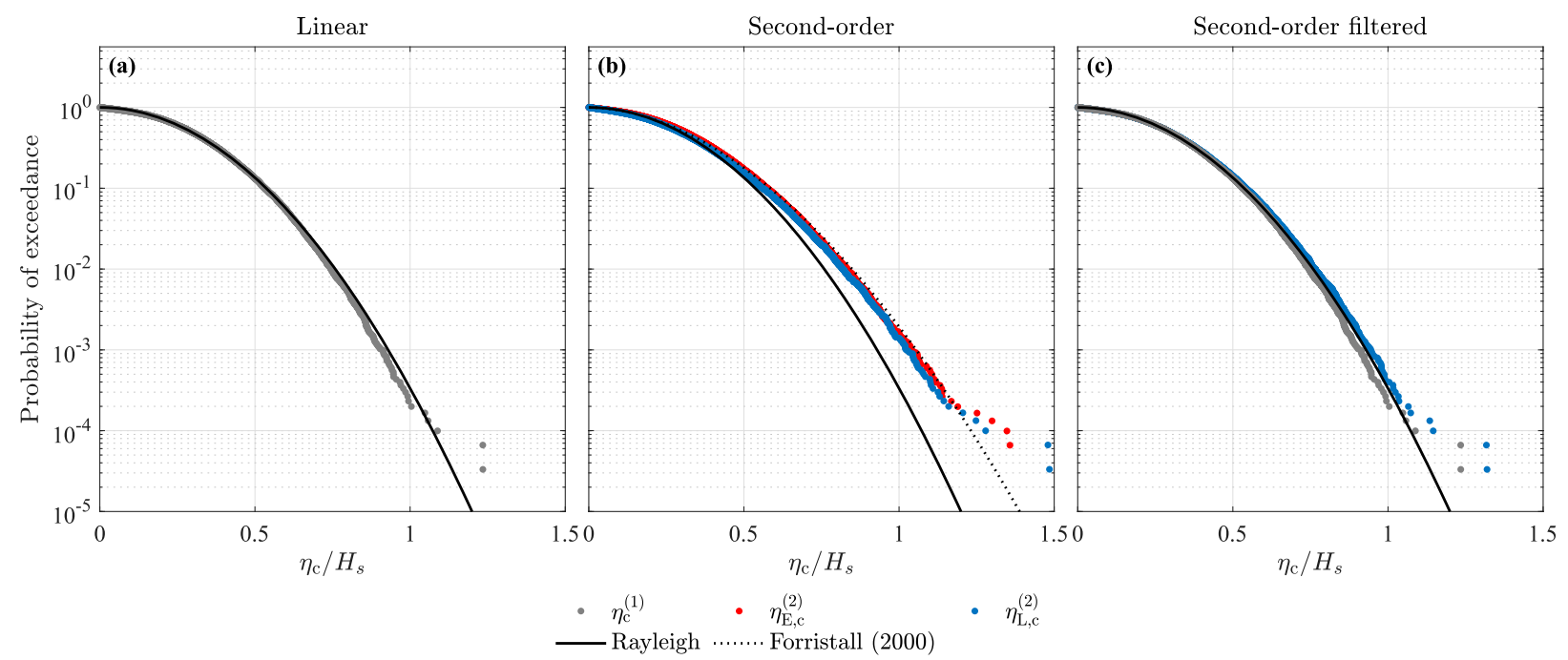

FIG. 2. (a)-(c) Crest height exceedance probability for random wave simulations carried out to illustrate the effects of second-order nonlinearity and filtering. Random linear waves simulated for a JONSWAP spectrum with $H_{s}=0.225 \mathrm{~m}, T_{p}=1.67 \mathrm{~s}, d=2 \mathrm{~m}, \sigma_{\theta}=10^{\circ}$ (see section 2) over 40 realizations with a duration of $1024 \mathrm{~s}$, giving $N \approx 30000$ waves. Gray, red, and blue markers denote individual linear, Eulerian second-order $\eta_{E, c}^{(2)}$ (Dalzell 1999), and Lagrangian second-order $\eta_{L, c}^{(2)}$ (Herbers and Janssen 2016) crest heights, respectively. The black solid and dotted lines show Rayleigh (1) and Forristall (2) crest height distributions, respectively. Shown in (c) is the effect of filtering $\left(0.37 f_{p}-6 f_{p}\right)$ the Lagrangian simulations.

large crests) is not significant when considering the Lagrangian measurement of steep directionally spread nonbreaking focused wave groups in the laboratory (McAllister and van den Bremer 2019).

An alternative explanation offered for the systematic underestimation of crest heights, which is consistent with in situ observations, is that the Lagrangian motion of a wave-following buoy cancels out second-order nonlinearity and essentially linearizes the resulting measurements (van Essen et al. 2018; Casas-Prat and Holthuijsen 2010). This is partially correct. The cancellation of second-order superharmonics that occurs as a result of Lagrangian motion is accompanied by an increase in second-order subharmonics, as identified by Srokosz and Longuet-Higgins (1986) and Herbers and Janssen (2016). Therefore, in principle, crest height measured by a wave-following buoy should not differ from that measured by an Eulerian observer as a result of second-order nonlinearity alone.

The linearization of buoy measurements occurs during the signal processing involved in converting from acceleration, which is measured by the buoy's instrumentation, to displacement (Marthinsen and Winterstein 1992; Seymour and Castel 1998; Prevosto et al. 2000; Forristall 2000). During this process, the calculated displacements are high-pass filtered. For Waverider buoys, the resulting low-frequency cutoff is $0.037 \mathrm{~Hz}$ (Datawell BV 2019), which represents a nonnegligible proportion of the bandwidth where second-order subharmonics lie. Hence, this process filters out the majority of the second-order contribution to crest height for ocean waves, for which typical peak frequencies $f_{p}$ lie at around $0.1 \mathrm{~Hz}$ and below, and the majority of second-order subharmonic energy lies at around $0-0.5 f_{p}$. Some more recent buoy designs also measure velocity through GPS-based Doppler velocimetry, which can improve low-frequency resolution (de Vries et al. 2003; Raghukumar et al. 2019). However, most off-the-shelf buoys measure displacements over a frequency band with a lower limit of around $0.037 \mathrm{~Hz}$.

Figure 2 illustrates the effects of second-order nonlinearity and filtering on crest height statistics. We simulate 40 directionally spread linear wave trains with a duration of $1024 \mathrm{~s}$ based on a JONSWAP spectrum with randomly assigned phase and direction (these wave conditions correspond to the experiments we present later; see section 2 for details). This gives a sample of approximately 30000 waves. The crest height distribution of these linear waves is presented in Fig. 2a, where each gray marker represents a single wave crest from our simulations. As is expected, these crests follow a Rayleigh distribution. We then calculate the corresponding secondorder bound components for each wave train using exact multicomponent second-order theory for both Eulerian (Dalzell 1999) and Lagrangian (Herbers and Janssen 2016) frames of reference. The red and blue markers in Fig. 2 show second-order Eulerian and Lagrangian crest heights, respectively. Both predictions of crest height follow the second-order crest height distribution proposed by Forristall (2000) well, and there is no significant difference between the predicted behavior of the two types of measurement. In Fig. 2c, blue markers show the 
crest heights for the bandpass filtered $\left(0.37 f_{p}-6 f_{p}\right)$ Lagrangian free surface elevation. After filtering, the Lagrangian crest height predictions follow the linear Rayleigh distribution and are essentially the same as the linear crests shown by the gray markers.

In previous work, we have experimentally examined the performance of a model wave-following buoy in steep directionally spread and crossing wave groups (McAllister and van den Bremer 2019). Under these conditions, we found that the buoy's behavior was essentially purely Lagrangian, and we did not observe any significant effects associated with the inertia or buoyancy of the model buoy. In those experiments, focused wave groups were examined, as their form provides an approximation to extreme wave groups that may be encountered in the ocean. For a random linear process, such as dispersively focusing water waves, the shape of extreme events will tend to the autocorrelation function of the underlying power spectrum (Lindgren 1970; Boccotti 1983). This concept referred to as NewWave (Tromans et al. 1991) or quasi-determinism (Boccotti 1983; Fedele and Tayfun 2009) has been validated for Eulerian measurements (Santo et al. 2013) and buoy measurements made in shallow water (Whittaker et al. 2016).

In this paper, we experientially examine whether for stochastic irregular wave trains measured Eulerian and buoy (Lagrangian) crests height statistics and sea-state parameters are comparable. We create sea states with different degrees of directional spreading including crossing. We then investigate the average measured extreme wave form of the irregular waves we create to further assess the applicability of the quasi-deterministic or NewWave approach to measurements made by buoys. Finally, we propose a method for retrospectively correcting in situ crest height distributions produced by wave-following buoys that effectively filter out bound subharmonic waves.

This paper is laid out as follows. First, in section 2 we outline our experimental method. We then present the results of our experiments in section 3 . We discuss how our findings may be used to reinterpret in situ buoy data in section 4, and draw conclusions in section 5 .

\section{Experimental method}

\section{a. Experimental setup}

The experiments presented herein were carried out in the FloWave Ocean Energy Research Facility at the University of Edinburgh. This is a circular wave basin, which is $2 \mathrm{~m}$ deep and surrounded by 168 actively absorbing force-feedback wavemakers, which allows for the creation of waves that travel in any direction.
TABLE 1. Gauge array, mean buoy positions, and the difference between the two.

\begin{tabular}{|c|c|c|c|c|c|c|}
\hline \multirow[b]{2}{*}{ No. } & \multicolumn{2}{|c|}{ Gauge } & \multicolumn{2}{|c|}{ Buoy } & \multicolumn{2}{|c|}{ Difference } \\
\hline & $x(\mathrm{~m})$ & $y(\mathrm{~m})$ & $x(\mathrm{~m})$ & $y(\mathrm{~m})$ & $\Delta x(\mathrm{~m})$ & $\Delta y(\mathrm{~m})$ \\
\hline 1 & -2.56 & 0.78 & -2.47 & 0.73 & 0.11 & 0.05 \\
\hline 2 & -1.28 & 0.08 & -1.18 & 0.07 & 0.1 & 0.01 \\
\hline 3 & -0.05 & 0.01 & 0 & 0 & 0.05 & 0.01 \\
\hline 4 & 1.16 & 0.09 & - & - & - & - \\
\hline 5 & 0.44 & 1.25 & 0.55 & 1.21 & 0.11 & 0.04 \\
\hline 6 & 1.85 & 0.82 & 1.84 & 0.81 & 0.01 & 0.01 \\
\hline 7 & 1.12 & -1.57 & 1.11 & -1.55 & 0.01 & 0.02 \\
\hline 8 & -0.75 & -1.75 & -0.70 & -1.76 & 0.05 & 0.01 \\
\hline
\end{tabular}

Eulerian measurements of free surface elevation were made using resistive wave gauges, which were calibrated at the start of each day of testing. Eight wave gauges were installed in the tank in the positions listed in Table 1 and shown in the schematic Fig. 3. We define the coordinate system where waves propagate in the positive $x$ direction, the angle $\theta$ is measured in the clockwise direction from the positive $x$ axis, and the position $x=0$, $y=0$ lies at the center of wave tank.

To perform a direct comparison between Eulerian and buoy measurements, each set of experiments we carried out was repeated with the wave gauges raised and model buoys placed in the same positions. Seven model buoys were located at the positions listed in Table 1 and indicated by black markers on the schematic Fig. 3. The buoy positions listed in Table 1 represent the mean position at which the buoys were located during experiments.

\section{MODEL BUOYS AND MOTION TRACKING}

We measure the position of our model buoys using a Qualisys motion tracking system, consisting of eight calibrated infrared cameras located around the circumference of the tank, which simultaneously track the position of the buoys. To aid their detection, the spherical model buoys were covered in a coating that reflects the specific band of infrared light emitted and recorded by the cameras. The buoy's position in each set of images is then triangulated to measure its global position within the tank.

To measure free surface elevation, wave-following buoys first measure acceleration, which is then integrated twice to give displacement. These displacements are projected onto the coordinate system of heave, north, and east displacements. In our experiments, we effectively assume that there is no error in this process [see van Essen et al. (2018) for a discussion of this assumption], and consider the position of our model buoy to be what a wave buoy in the ocean would measure.

The model buoys we use have a diameter $D=0.07 \mathrm{~m}$ and were designed to have a density approximately half 


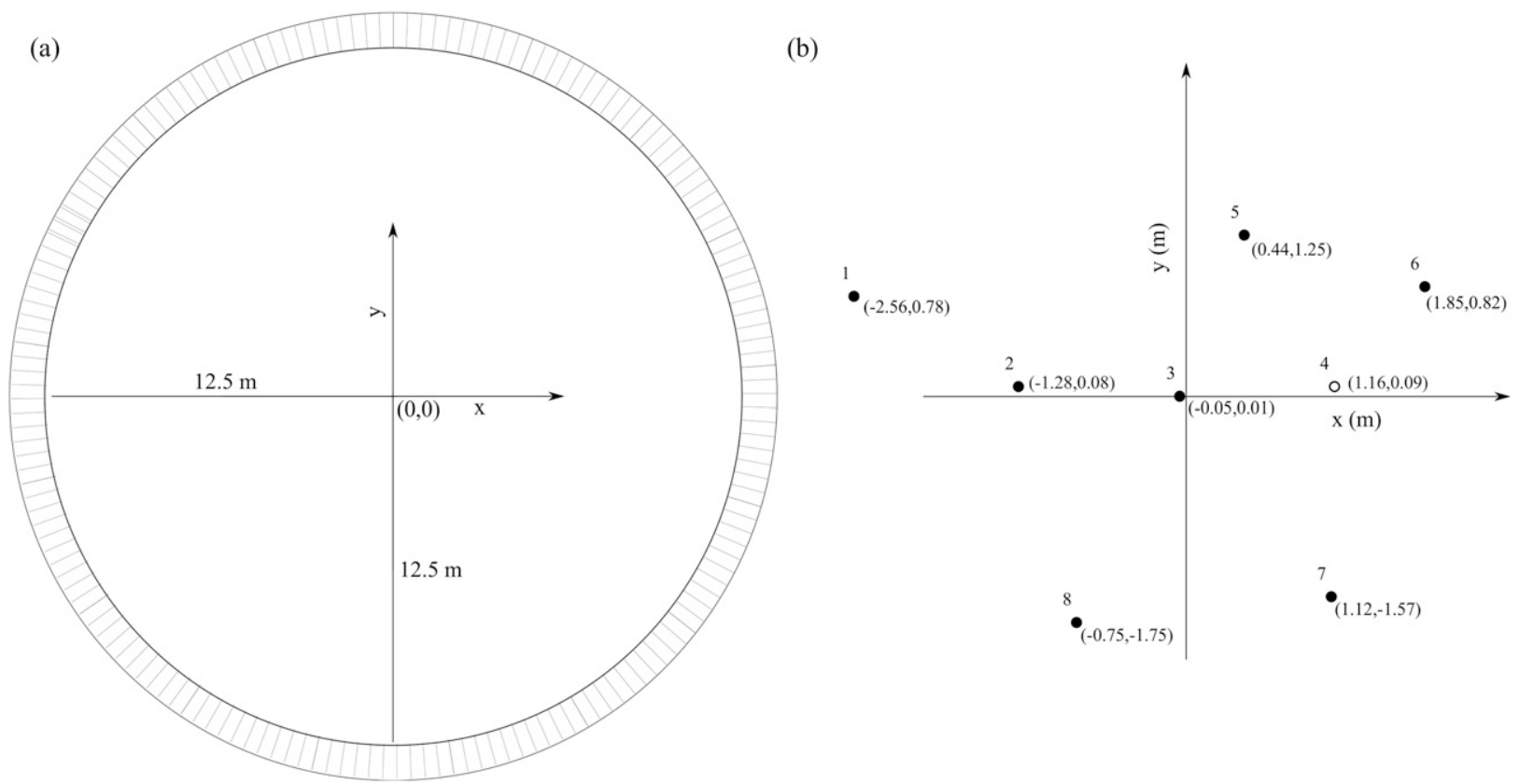

FIG. 3. Schematic diagram illustrating (a) tank layout and (b) array of buoy and wave gauge positions.

that of water. This size of the buoys was chosen to provide the best possible representation of wave-following buoy operating in the ocean, in other words, to maintain similar scaling parameters $D / \lambda_{0}$ and $a_{0} / D$, within the constraints of practicality in terms of measuring the position of the buoy. The ratio of buoy diameter to characteristic wavelength $D / \lambda_{0}$ for both full scale and our model buoys lie well below the limit $D / \lambda_{0} \ll 1$, meaning the effects of diffraction are not important. However, when considering the importance of inertial effects the ratio of characteristic wave amplitude to diameter $a_{0} / D$ of our buoy is somewhat lower than that of a real buoy. This means that our model may be slightly too large and more likely to exhibit inertial effects. However, in McAllister and van den Bremer (2019), which contains a detailed discussion of these scaling considerations, the same size of buoy did not show any observable inertial effects under similar conditions.

To maintain positioning at the desired location, each buoy was fixed to the floor of the tank using an auxiliary mooring. These simple taut moorings were designed to be highly flexible with the intention of having minimal effect measured free surface elevation. Previous experiments showed that this flexible auxiliary mooring configuration had no observable effect on the measured Lagrangian free surface elevation for individual wave groups (McAllister and van den Bremer 2019). We emphasize that the effect of a mooring on buoy motion in a realistic configuration is not the objective of the present study. However, for long-duration irregular wave trains some mooring effects are unavoidable as a result of Lagrangian-mean displacements that will accumulate over time.

\section{b. Matrix of experiments and input parameters}

The aim of our experiments is to produce and measure irregular waves representative of extreme ocean conditions. To achieve this, we base our input waves on the empirically derived JONSWAP spectrum (Hasselmann et al. 1973),

$$
\begin{aligned}
S(f)= & \beta g(2 \pi)^{-4} f_{p}^{-(5+n)} f^{n} \exp \left[\frac{n}{4}\left(\frac{f_{p}}{f}\right)^{-4}\right] \\
& \times \gamma^{\exp \left[-\left(f-f_{p}\right)^{2} / 2 \sigma^{2} f_{p}^{2}\right]}
\end{aligned}
$$

which is characteristic of fetch-limited unimodal sea states. The coefficient $\beta$ is a scaling parameter, which was selected to give the desired significant wave height $H_{s}$. For all of our experiments, the peak frequency $f_{p}$ was $0.6 \mathrm{~Hz}$; this frequency was chosen to optimize wave generation and absorption by the wavemakers, and ensure that resulting waves propagate on deep water $\left(k_{p} d=2.91\right)$. The shape parameters were defined using typical values: $n=-5, \gamma=3.3$, and $\sigma\left(f \leq f_{p}\right)=0.07$ and $\sigma\left(f>f_{p}\right)=0.09$.

To measure the true spectrum of a given sea state in the ocean, the duration of the observation $T$ would have to be infinitely long, hence any finite duration realization 
of this spectrum will not have the exact form of the underlying spectrum. To correctly reproduce this natural variability when prescribing amplitudes to discrete wave components in a wave tank, for each discrete amplitude, a value should be drawn from a normal distribution with variance $S(f)$. As we are interested in changes to spectral shape between equivalent Eulerian and buoy measurements, we define the amplitudes at each frequency deterministically $a(f)=\sqrt{2 S(f) \delta f}$, which does not capture this natural variability and can affect the resulting group structure of the resulting waves (Tucker et al. 1984).

The duration of each of our experiments was $T=1024 \mathrm{~s}$, which gives a frequency resolution of $\delta f=1 / 1024 \mathrm{~Hz}$. The wavemakers generate in the frequency band $0.2-2 \mathrm{~Hz}$, which allows for the creation of 1844 discrete wave components. To ensure that the waves we create within the tank are ergodic and spatially homogeneous using this limited number of waves, we implement directional spreading using a single summation method (Miles and Funke 1989),

$$
\eta(\mathbf{x}, t)=\sum_{n=1}^{N} a\left(f_{n}\right) \cos \left(\mathbf{k}_{n} \cdot \mathbf{x}-\omega_{n} t+\mu_{n}\right),
$$

where $\mathbf{k}_{n}=\left[k_{n} \cos \left(\theta_{n}\right), k_{n}\left(\theta_{n}\right)\right]$, and the absolute wavenumber $k_{n}=\left|\mathbf{k}_{n}\right|$ is related to frequency $f_{n}=\omega_{n} / 2 \pi$ by the linear dispersion relation $\omega_{n}=\sqrt{g k_{n} \tanh \left(k_{n} h\right)}$. The random phase shift $\mu_{n}$ is sampled from a uniform distribution between 0 and $2 \pi$. The desired directional spreading distribution is achieved by sampling the direction of each discrete wave component $\theta_{n}$ from a probability distribution of the same form. In our experiments, we aim to produce a wrapped normal spreading distribution centered around $\theta=\theta_{0}$,

$$
\Omega(\theta)=\frac{1}{\sqrt{2 \pi} \sigma_{\theta}} \sum_{n=-\infty}^{\infty} \exp \left[-\frac{\left(\theta-\theta_{0}+2 \pi n\right)^{2}}{2 \sigma_{\theta}^{2}}\right] .
$$

Therefore, each direction $\theta_{n}$ is randomly sampled from a normal distribution with characteristic spreading width $\sigma_{\theta}$. To generate crossing sea states, we sample $\theta_{n}$ from two superimposed normal distributions with mean directions separated by $\Delta \theta$.

We carry out experiments with input significant wave height $H_{s}=0.225 \mathrm{~m}$ for four different directional conditions (see Table 2): for following or unimodal conditions with characteristic spreading widths of $\sigma_{\theta}=10^{\circ}$, $20^{\circ}$, and $30^{\circ}$, and for crossing conditions with characteristic spreading width $\sigma_{\theta}=20^{\circ}$ and crossing angle $\Delta \theta=$ $90^{\circ}$. For each of these conditions, three repeat experiments with different random phases $\mu_{n}$ and directions $\theta_{n}$ were carried out. To investigate the importance of
TABLE 2. Test matrix.

\begin{tabular}{cccc}
\hline \hline$H_{s}$ & $\sigma_{\theta}\left({ }^{\circ}\right)$ & $\Delta \theta\left(^{\circ}\right)$ & Number of realizations \\
\hline 0.225 & $10,20,30$ & - & 3 \\
0.225 & 20 & 90 & 3 \\
0.175 & $10,20,30$ & - & 1 \\
\hline
\end{tabular}

steepness, three additional experiments were carried out for each following sea state at a reduced input significant wave height $H_{s}=0.175 \mathrm{~m}$, using the same random phases and directions as realizations carried out at $H_{s}=0.225 \mathrm{~m}$.

\section{c. Estimation of sea-state parameters}

Measurements made by buoys are often used to compute spectral parameters, such as significant wave height $H_{s}$ and peak period $T_{p}$. These summary statistics are commonly used to calibrate phase-averaged ocean forecast and hindcast models (Janssen et al. 1997; Bidlot et al. 2002) and to characterize environmental conditions across the oceans (Hasselmann et al. 1980; Pontes 1998; Mork et al. 2010; Fedele and Arena 2010; LeneeBluhm et al. 2011). To understand actual conditions achieved in the tank, we estimate the spectral parameters of the free surface elevation measured by the gauges. We also carry this out for our buoy measurements to examine if any difference exist between the two measurements types. We use the superscript $\star$ to denote estimated parameters.

We estimate spectral parameters by fitting a JONSWAP spectrum to our measurements following the method outlined by Young (2006). First, significant wave height $H_{s}^{\star}$ is estimated as $4 \sqrt{m_{0}}$, where $m_{0}$ is the zeroth moment of the measured spectrum. The peak period $T_{p}^{\star}=1 / f_{p}^{\star}$ is then estimated as

$$
f_{p}^{\star}=\frac{\int_{0}^{6 f_{p}} S(f)^{5} f d f}{\int_{0}^{6 f_{p}} S(f)^{5} d f} .
$$

To fit a JONSWAP spectrum to our measurements, first the slope of the high-frequency tail $n^{\star}$ is estimated over the range of frequencies $2 f_{p}^{\star}<f<6 f_{p}^{\star}$. The remaining parameters $\gamma^{\star}$ and $\sigma^{\star}$ are then fitted using the entire measured spectrum $\left(0<f<6 f_{p}^{\star}\right)$.

The time-domain parameters skewness $\lambda_{3}$ and kurtosis $\lambda_{4}$ are often used to quantify the nonlinearity of a given sea state and the likelihood of extreme waves. We also estimate these two parameters using both our Eulerian and buoy measurements to establish if any systematic differences exist between the two types of measurement. 


\section{Results}

\section{a. Filtering}

The signal processing that is carried out when buoys convert acceleration and velocity measurements to displacements, results in the high-pass filtering of the free surface elevation that they measure. For example, the resulting operational frequency band of a Datawell Waverider buoy is $0.037-0.64 \mathrm{~Hz}$. To replicate this with our experimental results, we filter our measured free surface elevations over the band $0.37 f_{p}<f<6 f_{p}$. This is based on the assumption that the average peak frequency of extreme ocean waves will be approximately $0.1 \mathrm{~Hz}$. Storm waves will often have frequencies lower than $0.1 \mathrm{~Hz}$; for such waves the effects of filtering will be even more pronounced, making this choice of frequency conservative.

\section{b. Sea-state parameters}

In Fig. 4, we compare the sea-state parameters measured by our model buoys to those measured by the array of Eulerian wave gauges. For each individual experiment, we take the mean across all of the available measurements ( 7 buoys and 8 gauges). In this figure, the black markers show parameters estimated using the unprocessed buoy data, and the red markers show the same parameters estimated using the buoy measurements after filtering. Error bars in these figures represent two standard deviations of the estimates made from the different measurements ( 7 buoys and 8 gauges) used to generate the mean values (see appendix $\mathrm{C}$ for a full discussion of sources of measurement error). We only provide error bars for the parameters estimated for the unfiltered data (black markers) and not the filtered data (red markers) to aid clarity.

(i) Significant wave height $H_{s}$ : The significant wave height measured by our model buoys (Fig. 4a) is consistently higher than the true value measured by the wave gauges. On average, the values estimated using the buoy measurements are $2.8 \%$ larger ( $\approx 5 \mathrm{~mm}$ at laboratory scale). This difference reduces slightly as the degree of spreading is increased; therefore, it may be a result of the forward transport of buoys induced by large waves, resulting in the observation of larger crests as the waves focus. Filtering the free surface elevation reduces this difference, as filtering the measurements essentially removes energy and reduces $m_{0}$.

(ii) Peak period $T_{p}$ : The peak period measured by our model buoys (Fig. 4b) is slightly larger than the true period measured by the wave gauges. However, the differences we observe are very small, on average $1 \%$, and not always significant. An increase in period is expected between Lagrangian and Eulerian frames of reference (Longuet-Higgins 1986). Stokes drift causes a Doppler shift in the period measured by a Lagrangian observer, which is proportional to the steepness of the waves measured and reduces with increasing directional spreading. There is no observable trend between the degree of spreading and the measured Lagrangian period.

(iii) JONSWAP parameters $n$ and $\gamma$ : The results of fitting a JONSWAP spectrum to our measurements, namely the parameters $n^{\star}$ (Fig. 4c) and $\gamma^{\star}$ (Fig. 4d) vary quite significantly across our buoys and gauges, as illustrated by the large error bars. Thus, for both parameters, it is hard to infer any trends with confidence. Ideally, to fit the exponent $n$ to the tail of the spectrum, several decades of data would be used, whereas we use the relatively short frequency range $2 f_{p}^{\star}<f<6 f_{p}$. We fit over this band, as it represents the band of frequencies measured by a Waverider buoy, assuming a full-scale peak frequency of approximately $0.1 \mathrm{~Hz}$. The slope of the high-frequency tail of the spectrum is steeper for the buoy measurements; this may be caused by the attenuation of high-frequency waves. At around $6 f_{p}$, the ratio of wavenumber to buoy radius $D k / 2 \approx 1$. At values of $D k / 2$ greater than unity, motion will be attenuated by the buoy. As the parameter $n^{\star}$ describes the tail of the spectrum, it is not affected by high-pass filtering.

(iv) Skewness $\lambda_{3}$ : The skewness of the free surface elevation measured by our model buoys and wave gauges (Fig. 4e) agree reasonably well. For low degrees of spreading, the free surface elevation measured by the buoys exhibits slightly increased skewness, which is consistent with the hypothesis in item i, that forward transport by large waves may cause a buoy to spend more time at the crest of waves. Filtering the buoy measurements significantly reduces the skewness of the free surface elevation, bringing it close to zero, as subharmonic bound waves are removed.

(v) Kurtosis $\lambda_{4}$ : The kurtosis of the free surface elevation measured by our model buoys (Fig. 4f) is slightly larger than that measured by the wave gauges. This difference is larger at low degrees of spreading. Filtering the buoy measurements also slightly reduces their kurtosis.

\section{c. Crest height distributions}

Ultimately, it is wave crests and their likelihood that inform the design of fixed offshore structures. We examine the distribution of wave crests measured during our experiments in Fig. 5. Here, a wave crest is defined 

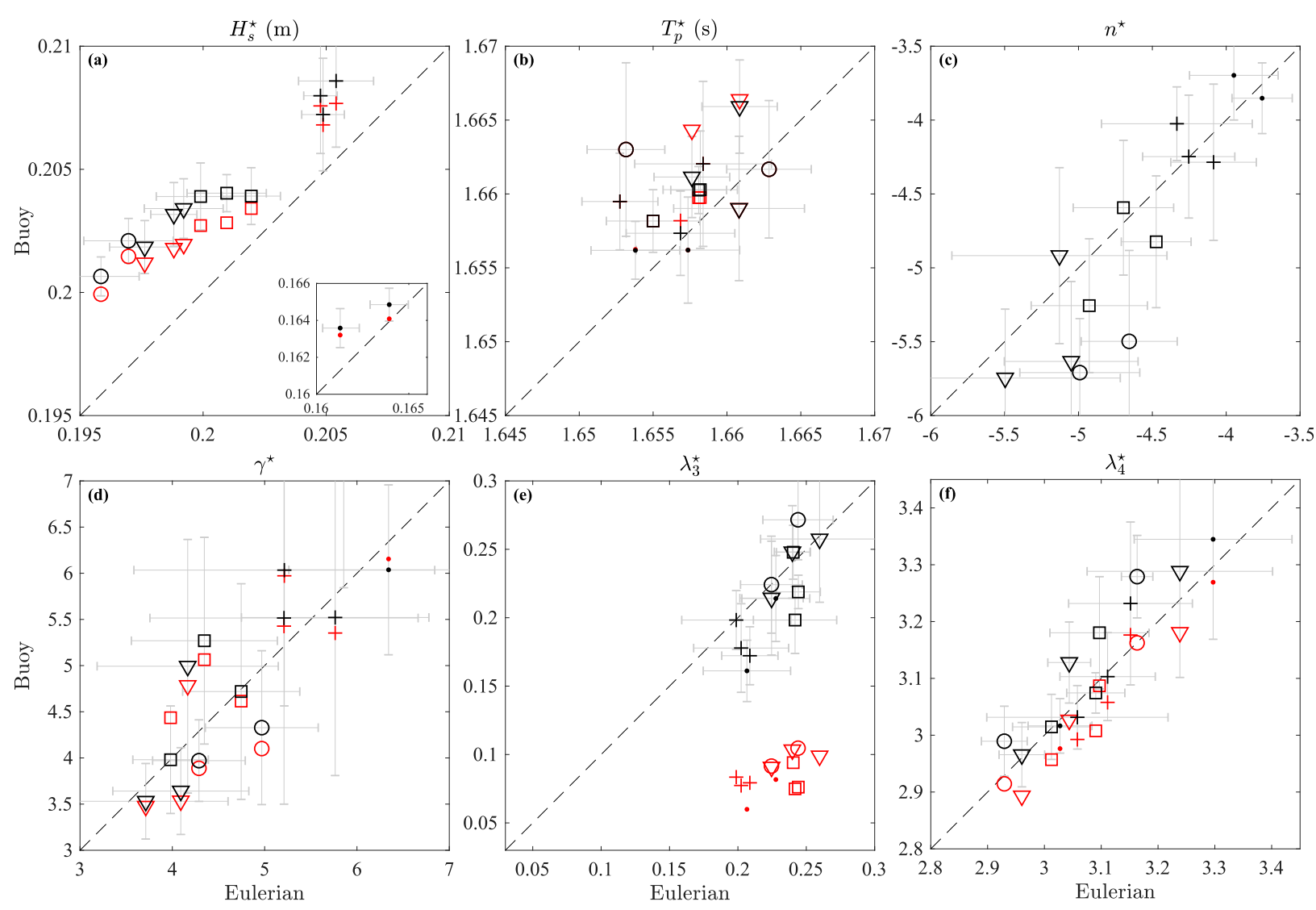

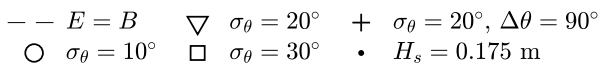

FIG. 4. Comparison of sea-state parameters estimated using buoy and Eulerian wave gauge measurements. The black markers show estimated parameters across the different spreading conditions we test, and the red markers show the same parameters estimated after filtering the buoy measurements $\left(0.37 f_{p}-6 f_{p}\right)$. The dot markers denote experiments carried out at reduced steepness (see Table 2).

as the maximum value of free surface elevation between successive up- and downcrossings. In Fig. 5, we present crest heights measured by buoy and gauge pair 3, located at the center of the tank $(x=0, y=0)$. In each subfigure, data measured over three realizations of the same input spreading conditions are presented. The same input significant wave height and peak period were used for all four sets of experiments with different spreading conditions. The distribution of crest heights we measure for both buoys and gauges is slightly under predicted by the second-order Forristall distribution (2). The waves we create are very steep and at times breaking. Similar deviations from second-order theory are also observed for steep sea states in Onorato et al. (2009) and Latheef and Swan (2013). Moving from left to right in Fig. 5, as the degree of spreading is increased, the measured crest heights tend toward the second-order distribution. Between the crest heights measured by the buoy and the gauge, there are some differences. For the gauge-buoy pair we present, the crests measured by the wave gauge appear to be slightly larger. However, the difference is not significant. In Fig. 6, we combine crest heights measured across all 7 buoy and gauge pairs; increasing the number of observations removes any noticeable difference between the two sets of measurements. It should be noted that combining concurrent measurements will affect the likelihood of observing a given crest height, as although the observations are made at different locations, they are correlated. Thus, we only include theoretical distributions in Fig. 6 for reference.

\section{d. Wave height distributions}

It is well established that crest-to-trough wave height is not affected by second-order nonlinearity (LonguetHiggins 1980). Therefore, in principle, wave heights measured by buoys and gauges should be the same even after filtering. We examine the distribution of wave heights measured during our experiments in Fig. 7. Here, wave height is defined using the maximum and 

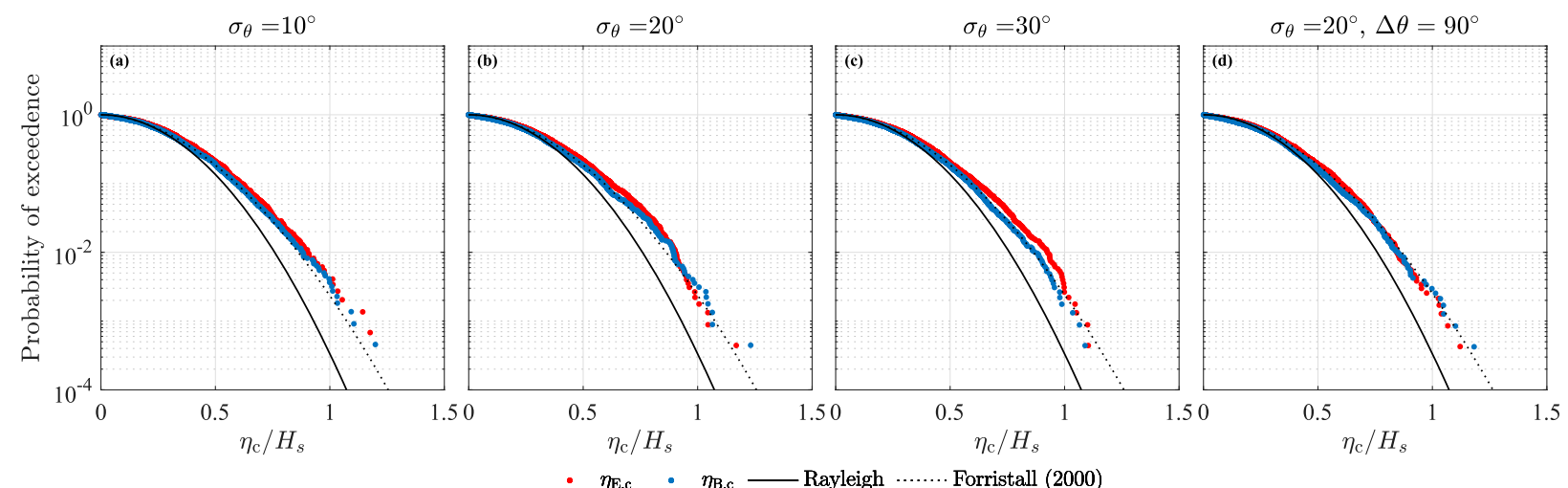

FIG. 5. Crest height exceedance probability measured during experiments. Red and blue markers show individual crest heights measured by wave gauge $\left(\eta_{E, c}\right)$ and buoy $\left(\eta_{B, c}\right)$ pair 3, located at $x=0, y=0$. The black solid and dotted lines show Rayleigh (1) and Forristall (2) distributions, respectively. (a)-(c) Moving from left to right, the degree of directional spreading is increased, and (d) a crossing sea state is shown.

minimum values of free surface elevation between successive upcrossings. In Fig. 7, we present wave heights measured by buoy and gauge pair number 3 , located at the center of the tank $(x=0, y=0)$. In each subfigure, data measured over three realizations of the same input spreading conditions are presented. The same input significant wave height and peak period were used for all four sets of experiments.

When considering wave height, our measurements made using buoys and wave gauges agree very well and are essentially the same. The distribution of wave heights we measure for both buoys and gauges is slightly under predicted by the bandwidth-corrected Rayleigh distribution (3). This suggests that there are nonlinear effects beyond second-order that are increasing the height of the waves we have created. We also included the Rayleigh distribution of wave heights proposed
Longuet-Higgins (1952) for narrow-banded conditions [cf. (3) with $\Psi=0$ ],

$$
P\left(h_{u}>h\right)=\exp \left[-2\left(\frac{h}{H_{s}}\right)^{2}\right] .
$$

In Fig. 7, if bandwidth is not considered, it appears that the wave heights follow a linear distribution. However, this is misleading as comparison with the finite bandwidth in (3) shows that nonlinearity beyond secondorder is causing an increase in measured wave height.

\section{e. Average wave form}

The theory of quasi-determinism that underpins nonlinear crest and wave height statistics implies that the average wave form of extreme waves within given sea state follows the form of the autocorrelation function of
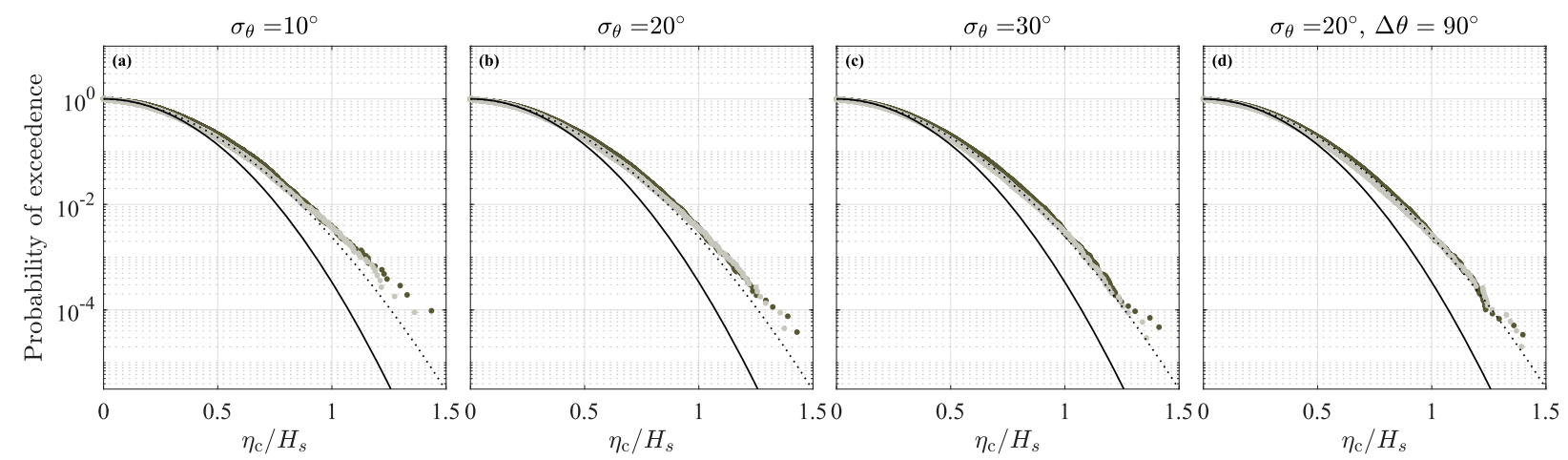

- $\eta_{\mathrm{E}, \mathrm{c}} \quad \eta_{\mathrm{B}, \mathrm{c}}-$ Rayleigh …...... Forristall (2000)

FIG. 6. Crest height exceedance probability, measured during experiments. Black and gray markers show individual crest heights measured across all wave gauges $\left(\eta_{E, c}\right)$ and buoys $\left(\eta_{B, c}\right)$. The black solid and dotted lines show Rayleigh (1) and Forristall (2) distributions, respectively. (a)-(c) Moving from left to right, the degree of directional spreading is increased, and (d) a crossing sea state is shown. (Figure 5 shows the same information, but only for pair 3.) 

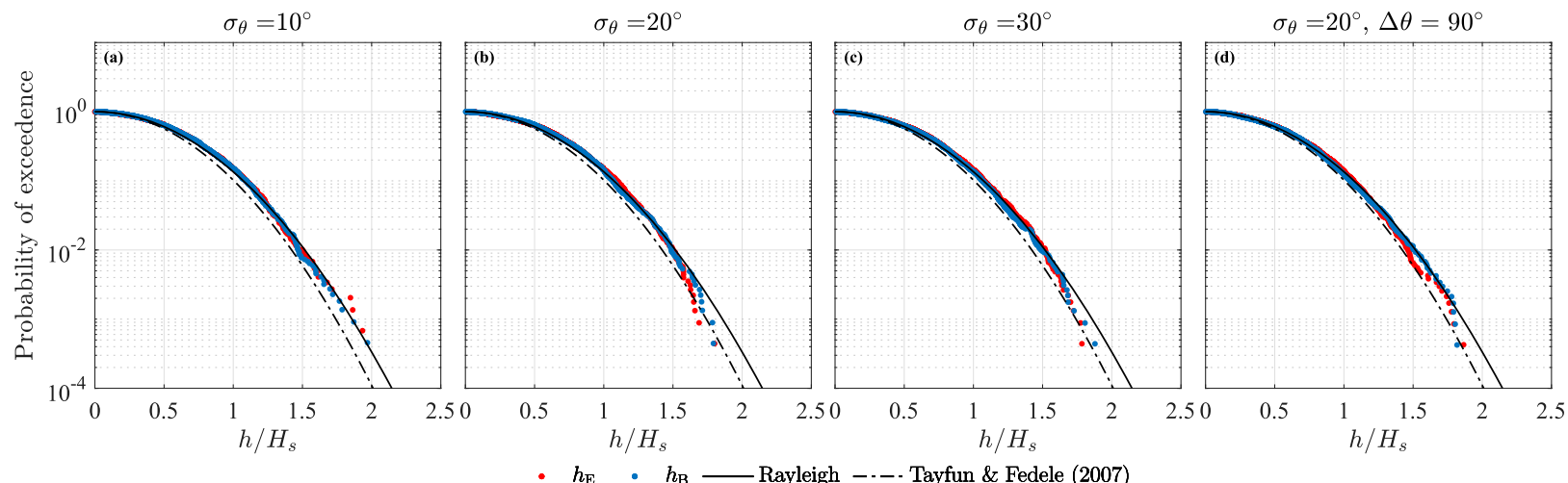

FIG. 7. Wave height exceedance probability, measured during experiments. Red and blue markers show individual wave heights measured by wave gauge $\left(h_{E}\right)$ and buoy $\left(h_{B}\right)$ pair 3, located at $x=0, y=0$. The black solid and dot dashed lines show Rayleigh (1) and bandwidth-corrected Rayleigh [(3); Tayfun and Fedele 2007] distributions, respectively. (a)-(c) Moving from left to right, the degree of directional spreading is increased, and (d) a crossing sea state is shown.

the underlying spectrum (Lindgren 1970; Boccotti 1983). In other words, the average form of extreme waves occurring in a random sea state may be approximated by a focused wave group based on the underlying spectrum. To assess the validity of this assumption for buoy measurements, we examine the average wave form, and corresponding second-order bound components, of the largest waves examined during our experiments.

For each sea state, we select the 20 largest crests and troughs, which are normalized and then averaged, giving the average wave form for an extreme crest $\bar{\eta}_{c}$ and trough $\bar{\eta}_{t}$. Through addition of these two wave forms, it is possible to extract an approximation to the average linear wave form and resulting bound waves (Whittaker et al. 2016),

$$
\bar{\eta}_{\mathrm{odd}}=\frac{\bar{\eta}_{c}-\bar{\eta}_{t}}{2}, \text { and } \quad \bar{\eta}_{\mathrm{even}}=\frac{\bar{\eta}_{c}+\bar{\eta}_{t}}{2}
$$

The subscripts odd and even denote wave components of odd and even powers in steepness when considering free surface elevation as a Stokes-type expansion.

The average linear wave form (Figs. 8a-f), subharmonics (Figs. 8g-1), and superharmonics (Figs. 8m-r) extracted from our measurements are shown by the black lines in Fig. 8. The blue and red lines in Figs. 8a-f show the scaled autocorrelation function of each sea state, and the sub- and superharmonics based on the scaled autocorrelation function calculated using Dalzell (1999) (gauges) and Herbers and Janssen (2016) (buoys) are shown in Figs. $8 \mathrm{~g}-1$ and $8 \mathrm{~m}-\mathrm{r}$, respectively. We introduce the notation $\eta_{T}$, to denote theoretical predictions based upon the autocorrelation function of surface elevation.

For both buoy and gauge measurements, the scaled autocorrelation function provides a good approximation to the average linear wave form of extreme events. Both sets of measurements exhibit slight horizontal asymmetry, where the wave preceding the maximum crest is slightly smaller than the one proceeding it. The period and crestfront steepness of the average wave form measured by the buoys is slightly reduced in comparison to the gauge measurements. The averaged second-order bound waves follow the theoretical predictions well, considering the approximate extraction method we use. For the buoy measurements, we observe the formation of a large setup of the subharmonics and cancellation of the superharmonics as predicted for Lagrangian motion. For our Eulerian gauges, we observe a small setdown of the subharmonics and large superharmonics. Hence, the effects illustrated deterministically in McAllister and van den Bremer (2019), can be readily applied to extreme waves measured within random sea states by both buoys and Eulerian devices.

\section{Buoy filtering reconstruction method}

The experiments presented herein have illustrated that a wave-following buoy's motion itself should not lead to the systematic underestimation of wave crest heights that is often observed for in situ measurements (see Fig. 1). We do not consider how measurements may be affected by a mooring, which may play a significant role and will be investigated in future work. We note that, if the effects of a realistic mooring were to influence the measurement of extreme crest, one might expect this to affect the distribution wave heights too. In light of our observations, we believe that the most likely cause of this systematic underestimation is filtering, as also suggested in Marthinsen and Winterstein (1992), Seymour and Castel (1998), Prevosto et al. (2000), and Forristall (2000).

Seymour and Castel (1998) present a simple method by which the true maximum crest height measured by a 

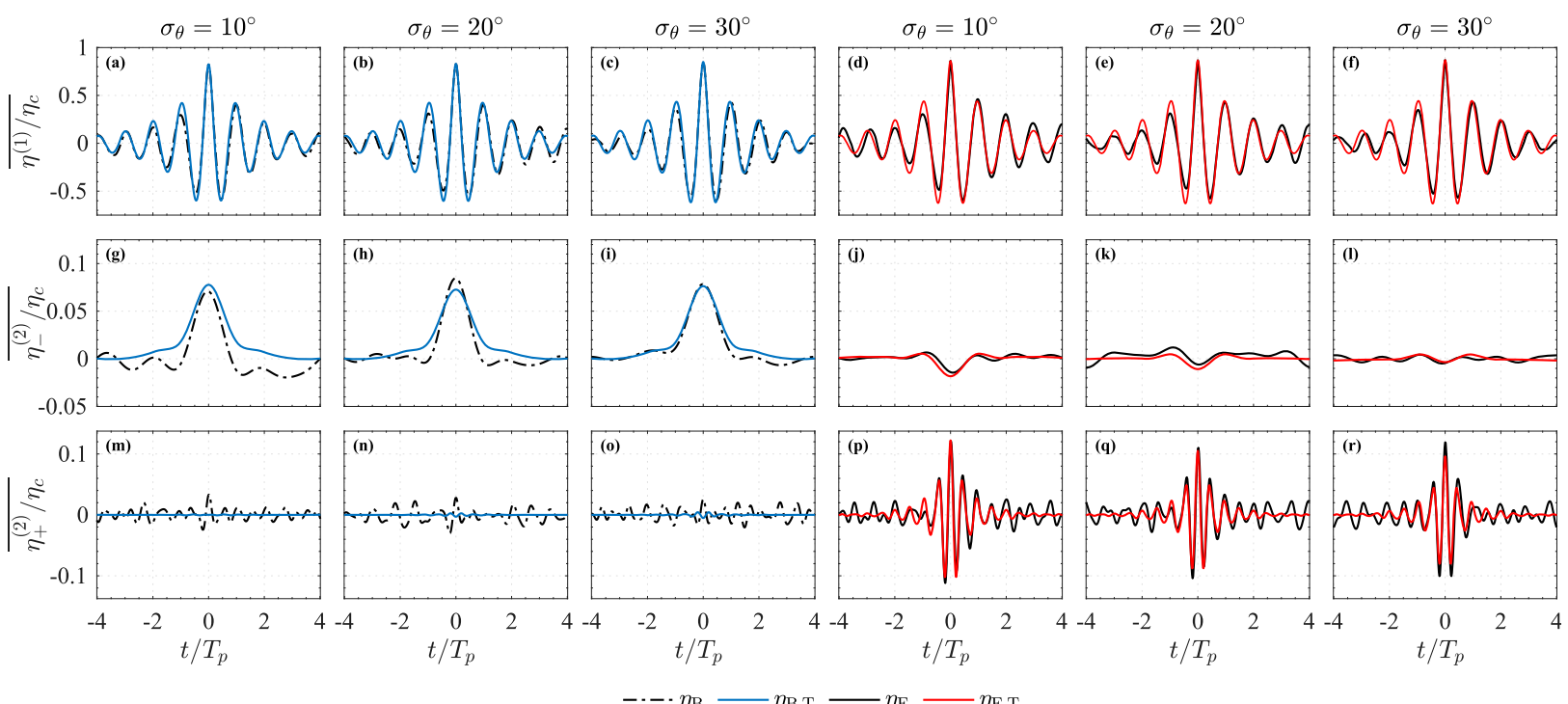

FIG. 8. Average extreme wave form measured by buoys and wave gauges, normalized by maximum crest height. (a)-(f) The linearized wave form, $(\mathrm{g})-(\mathrm{l})$ the second-order subharmonics $(-)$, and $(\mathrm{m})-(\mathrm{r})$ the second-order superharmonics $(+)$. Black lines show measurements, and colored lines the scaled autocorrelation functions of the underlying spectra and resulting second-order sub- and superharmonics calculated using exact Eulerian (Dalzell 1999) and Lagrangian (Herbers and Janssen 2016) theory.

buoy may be estimated. This method involves a process of reverse filtering, then performing an approximate conversion to a Eulerian frame of reference. After applying this approximate correction method to in situ buoy measurements, they suggest that for sea states of steepness $k_{p} H_{s}>0.1$, a $20 \%$ correction should be applied to maximum crest height $\eta_{c} / H_{s}$.

We have illustrated that the second-order contribution to crest height, or to the skewness of the free surface elevation, is the same for Lagrangian and Eulerian observers, as highlighted by Srokosz and Longuet-Higgins (1986). Therefore, it is unnecessary to convert buoy records into an Eulerian frame of reference to correct crest heights, as suggested by Seymour and Castel (1998). Moreover, reverse filtering in the frequency domain may not be possible for surface elevation records at frequencies where no phase or amplitude information exists.

In section 3, we have illustrated that the second-order contribution to extreme crests measured by a buoy can be well approximated using those associated with a focused wave group based on the autocorrelation function of linear surface elevation. In McAllister and van den Bremer (2019), we derived simplified closed-form expressions to evaluate the second-order contribution to crest height recorded by a Lagrangian observer at the point of focus for extreme wave groups. Making use of a narrow-banded approximation in frequency, but allowing for general directional spreading conditions, we obtain the following expressions for the second-order subharmonic contribution to crest height for a single directionally spread wave group,

$$
\tilde{\eta}_{L}^{(2)}\left(\sigma_{\theta}\right)=\tilde{\eta}_{E, \mathrm{SD}}^{(2)}+\frac{1}{2}\left(1+e^{-\sigma_{\theta}^{2}}\right),
$$

and two crossing wave groups,

$$
\tilde{\eta}_{L}^{(2)}(\Delta \theta)=\tilde{\eta}_{E, \mathrm{SD}}^{(2)}+\frac{1}{2}+\frac{1}{4}[1+\cos (\Delta \theta)]
$$

where $\tilde{\eta}^{(2)} \equiv \eta^{(2)} /\left(a_{0}^{2} k_{0} / 2\right)$ with $a_{0}$ and $k_{0}$ as the characteristic amplitude and wavenumber of the linear wave group at focus. In deep water, the Eulerian subharmonic setdown contribution to crest height $\tilde{\eta}_{E S \mathrm{SD}}^{(2)}$ is small and can be ignored $\left(\tilde{\eta}_{E, \mathrm{SD}}^{(2)}=-0.17\right.$ for $k_{0} d=3$ in unidirectional conditions and will be smaller for large directional spreading). It should be noted that $\sigma_{\theta}$ which we use to define the spreading width of our experiments in (6) corresponds to width of the energy spectrum, whereas $\sigma_{\theta}$ in (10) corresponds to the width of the amplitude distribution. Under the framework of quasi-determinism extreme waves will have an amplitude distribution based on the underlying energy spectrum. Hence, in this case, the definitions of $\sigma_{\theta}$ in both equations are consistent.

We now investigate using these expressions as a retrospective method to remove the effects of filtering from crest heights measured by buoys. The method we propose relies upon the assumption that the wave crests observed by a buoy $\eta_{c}$ are approximately linear $\left(\eta_{c} \approx \eta_{c}^{(1)}\right)$ owing to filtering, and can be retrospectively corrected 


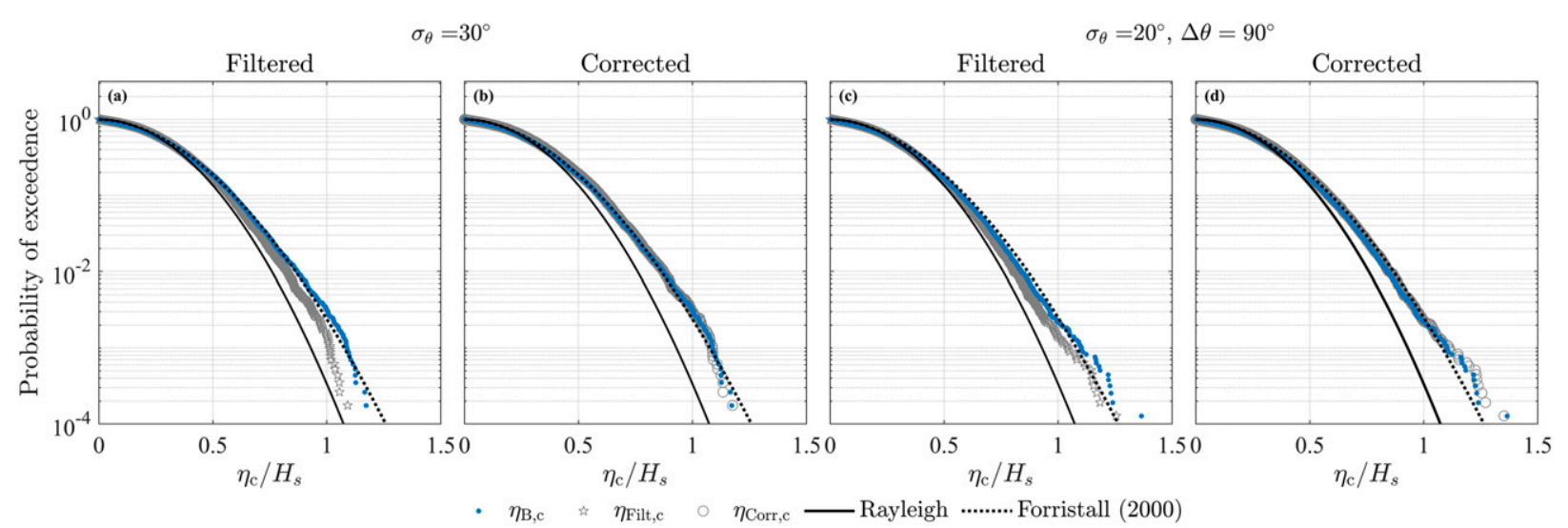

FIG. 9. Reconstructed crest height exceedance probability measured during two experiments for (a),(b) following and (c),(d) crossing sea states. Blue markers show individual crest heights measured by all seven buoys across all realizations. The black solid and dotted lines show Rayleigh (1) and Forristall (5) distributions, respectively. In (a) and (c), gray stars show the crest heights after filtering $\left(0.37 f_{p}-6 f_{p}\right)$ the buoy measurements, and in (b) and (d), gray circles show the same crest heights corrected using the proposed buoy filtering reconstruction method in (14).

through the addition of the second-order subharmonic components,

$$
\eta_{\mathrm{Corr}, c}=\eta_{c}+\eta_{c-}^{(2)}
$$

The contribution to crest height made by second-order subharmonics $\eta_{c^{-}}^{(2)}$ is then calculated using (10) or (11) for the given directional conditions,

$$
\eta_{c-}^{(2)}=\frac{1}{2}\left(\eta_{c}\right)^{2} k_{0} \tilde{\eta}^{(2)}\left(\sigma_{\theta}, \Delta \theta\right)\left(1-\varepsilon_{\mathrm{hpf}}\right),
$$

where we have dimensionalized (10) and (11) with the local linear crest amplitude $\eta_{c}$ and the wavenumber $k_{p}$, effectively making the assumption each crest is a local maximum (cf. theory of quasi-determinism). The parameter $\varepsilon_{\text {hpf }}$ is an ad hoc correction factor that accounts the for relative frequency at which the measurements are filtered, as this affects the amount of second-order contribution to crest height that is removed. For extratropical storms, the majority of subharmonic energy lies between 0 and $0.5 f_{p}$ [see McAllister et al. (2019) for an example where this is not the case]. However, the total second-order subharmonic contribution to crest height extends to frequencies higher than $0.5 f_{p}$. For extreme waves based on the JONSWAP spectra we examine, this frequency band ranges from 0 to approximately $2 f_{p}$. Therefore, the exact amount of second-order subharmonic energy removed by filtering and the required correction depends on the relative frequency cutoff frequency $f_{c} / f_{p}$. We define the parameter $\varepsilon_{\text {hpf }}$ empirically using exact second-order theory for a Lagrangian observer, where $\varepsilon_{\text {hpf }}$ represents the ratio energy being removed from the data to the total second-order subharmonic contribution, and estimate the relationship,

$$
\varepsilon_{\mathrm{hpf}}=e^{-2 f_{c} / f_{p}} .
$$

This ad hoc correction factor is not significantly affected by directional spreading, and appropriate for conditions with spectra that are relativity broadbanded $(\gamma \approx 3.3)$ (see appendix D for further details).

Figure 9 shows filtered $\eta_{\text {Filt,c }}$ (Figs. 9a,c), and then corrected $\eta_{\text {Corr }, c}$ (Figs. 9b,d) buoy measurements from our experiments using the proposed filtering reconstruction method. In this figure, measured wave crests are taken from all seven buoys and all three realizations of each sea state. The approximate correction works well, and the corrected measurements match the original unfiltered data for both following (Figs. 9a,b) and crossing (Figs. 9c,d) sea states.

\section{Conclusions}

Wave buoys are commonly considered to underestimate and effectively linearize extreme crest heights. We examine the nature of this phenomenon. Through stochastic calculations based on exact second-order theory (Dalzell 1999; Herbers and Janssen 2016), we show that crest heights measured by Eulerian and Lagrangian (buoy) sensors should not differ significantly. To confirm this is also the case for a partially submerged wavefollowing buoy in steep directionally spread irregular waves, we have carried out a new series of experiments.

In general, the wave-averaged sea-state parameters measured by our buoys do not vary significantly from the true values measured by the Eulerian wave gauges. The significant wave height $H_{s}$ and peak period $T_{p}$ measured by the buoys are approximately $2.8 \%$ and $1 \%$ larger, 
respectively. Filtering the buoy measurements, in a manner representative of the process carried out by buoys when converting measured accelerations to displacements, slightly reduces the measured significant wave height and kurtosis $\lambda_{4}$, and quite significantly affects the skewness $\lambda_{3}$ of the measurements.

The crest and wave height distributions measured by both our buoys and gauges follow our theoretical predictions and do not exhibit significant differences. The average linear wave form of the extreme waves in our measurements is well approximated by the autocorrelation function of their underlying spectra. The corresponding second-order sub- and superharmonics are also well approximated using secondorder calculations based on these autocorrelation functions.

We propose an approximate means of correcting crest heights measured by buoys that have been filtered. We apply this approximate correction to our experiments and show it is capable of retrospectively accounting for the effects of filtering and correcting in situ buoy measurements, both in directionally spread and crossing sea states.

Acknowledgments. This project was funded by the EPSRC and Wave Energy Scotland (grant no. 24840078 and 24841554). TSvdB was supported by a Royal Academy of Engineering Research Fellowship. The authors thank Dr. S. Draycott and Mr. E. Nixon, Dr. R. Gabl, and Dr. T. Davey at the FloWave Ocean Energy Research Facility for their assistance in planning and carrying out the experiments.

\section{APPENDIX A}

\section{Buoy Data}

The data in Fig. 1 were obtained from a single buoy within CDIP's network of buoys. The buoy's mooring $\left(33^{\circ} 13^{\prime} 29^{\prime \prime} \mathrm{N}, 119^{\circ} 52^{\prime} 54^{\prime \prime} \mathrm{W}\right)$ lies to the west of San Nicholas Island, which is located 61 miles from the Californian Coast. This particular buoy was chosen, as it is situated in deep water $\left(d=274 \mathrm{~m}\right.$ and $k_{p} d \approx 10$ with $k_{p}$ as the peak wavenumber) far from the coast, in an area exposed to large waves. Of the available data, five 30-min duration measurements were selected (see Table A1), giving a sample of $N=1066$ waves and hence similar probability levels to the waves created in our experiments. Measurements were first selected based on their steepness, then their spectral characteristics in both frequency and direction (peak period $T_{p}$ and direction $\theta_{p}$ ) to ensure they individual wave crests were samples from similar sea states.
TABLE A1. CDIP data from station 67 (San Nicholas Island), 30-min duration measurements recorded by a Waverider buoy on a depth of $274 \mathrm{~m}$.

\begin{tabular}{cccrc}
\hline \hline Date & Time (UTC) & $H_{s}(\mathrm{~m})$ & $T_{p}(\mathrm{~s})$ & $\theta_{p}\left({ }^{\circ}\right)$ \\
\hline 28 Dec 2006 & 0000 & 5.9 & 9.88 & 301 \\
28 Dec 2006 & 0100 & 6.85 & 10.53 & 304 \\
16 Nov 2015 & 1154 & 6.53 & 10.53 & 306 \\
16 Nov 2015 & 1224 & 6.77 & 10.53 & 302 \\
16 Nov 2015 & 1324 & 6.31 & 9.88 & 312 \\
\hline
\end{tabular}

\section{APPENDIX B}

\section{Second-Order Exceedance Probability}

The Weibull coefficients $\alpha$ and $\beta$ in (2) are related to the steepness parameter,

$$
S_{1}=\frac{2 \pi}{g} \frac{H_{s}}{T_{1}^{2}}
$$

and Ursell number,

$$
U_{r}=\frac{H_{s}}{k_{1}^{2} d^{3}}
$$

by the empirical relations

$$
\begin{aligned}
& \alpha_{2}=0.3736+0.2892 S_{1}+0.1060 U_{r}, \\
& \beta_{2}=2-2.1597 S_{1}+0.0968 U_{r}^{2},
\end{aligned}
$$

for $2 \mathrm{D}$ waves, and

$$
\begin{aligned}
& \alpha_{3}=0.3736+0.2568 S_{1}+0.0800 U_{r}, \\
& \beta_{3}=2-1.7912 S_{1}-0.5302 U_{r}+0.248 U_{r}^{2},
\end{aligned}
$$

for 3D waves (Forristall 2000).

\section{APPENDIX C}

\section{Measurement Error}

To quantify the sources of error affecting our comparison between Eulerian and buoy measurements, we examine the role of the various sources of error and uncertainty involved in our experiments.

For Eulerian measurements, the leading source of error is associated with wave gauge calibration and is approximately $0.4 \mathrm{~mm}$ (two standard deviations). Of the eight cameras, each possible pairing is used to measure the buoys' location; the residual values measure the time varying error in the position of the buoys and ranged from \pm 0.5 to $\pm 1.5 \mathrm{~mm}$. 


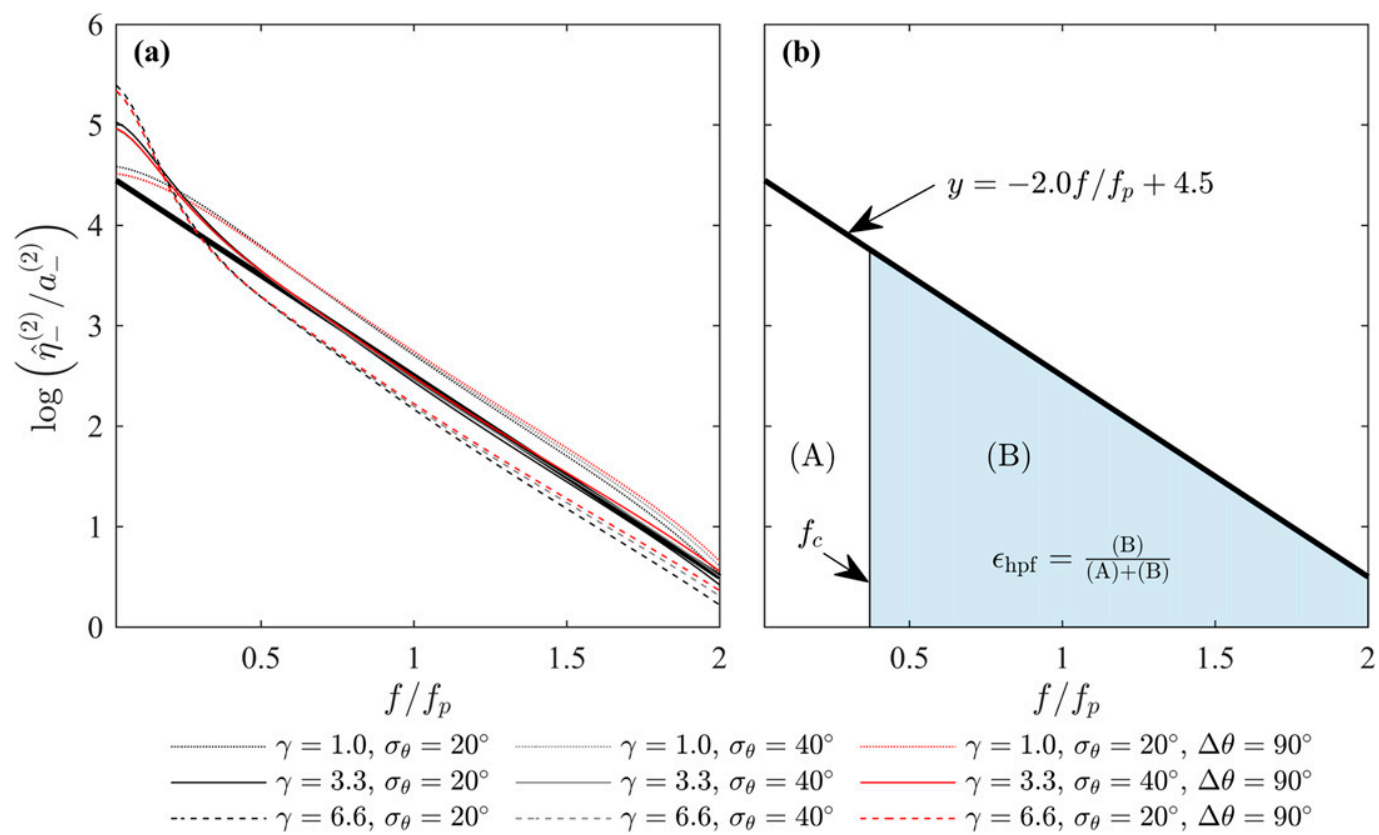

FIG. D1 . Estimation of the ad hoc correction factor $\varepsilon_{\text {hpf }}$. (a) The normalized log scale amplitude distribution of second-order subharmonics for Lagrangian measurement of focused wave groups based on JONSWAP spectra with peak enhancement factors $\gamma=1,3.3$, and 6.6, and for following $\left(\sigma_{\theta}=20^{\circ}\right.$ and $\left.40^{\circ}\right)$ and crossing $\left(\sigma_{\theta}=20^{\circ}, \Delta \theta=\right.$ $90^{\circ}$ ) directional conditions; the thick black line shows the curve fitted to this data, noting that only the gradient of the line affects (D2). (b) An illustration of how this fitted curve is used to estimate the parameter $\varepsilon_{\mathrm{hpf}}$ as a function of $f_{c}$, where the area labeled A represents the subharmonics that are removed and the area labeled B represents those that are retained after filtering.

Additional to sources of measurement error, spatial inhomogeneity and small variations in the construction of the buoys may also introduce error. To estimate these sources of error, we compute the energy measured at each wave gauge and for each buoy. We then estimate the variation in energy across the tank as the normalized standard deviation of energy across all gauges or buoys for a single experiment. The variation in energy, averaged over all of our experiments, measured across the tank by the wave gauges is $3.4 \%$, and between the model buoys is $5.4 \%$ (two standard deviations). To draw comparison between gauge and buoy pairs, it is important that they are located at the same position, and any misalignment may also cause error. However, as the buoys have a degree of freedom to move horizontally some misalignment during experiments is unavoidable. The error between the mean position of our model buoys and wave gauges is given in Table 1 and ranges from 0.11 to $0.1 \mathrm{~m}$.

\section{APPENDIX D}

\section{Relative Filtering Frequency Parameter $\varepsilon_{\text {hpf }}$}

Depending on the peak frequency $f_{p}$ at which a given sea state occurs, the relative high-pass-filter frequency cutoff $f_{c} / f_{p}$ changes. This relative cutoff frequency determines how much of the second-order subharmonic contribution to crest height is removed by filtering; that is, if $f_{c} \ll f_{p}$ the effect of filtering will be less significant. Hence, the retrospective correction we make to measured crest heights must account for this. Therefore, we seek a correction factor to account for the amount of second-order subharmonic contribution to crest height that is filtered out for a given ratio of $f_{c} / f_{p}$. To do so we use exact Lagrangian second-order theory (Herbers and Janssen 2016).

Figure D1a shows the normalized amplitude distribution of second-order subharmonics $\hat{\eta}_{-}^{(2)} / a_{-}^{(2)}$ as a function of normalized frequency, where $a_{-}^{(2)}$ is the amplitude of second-order subharmonics at focus. Calculations were carried out for wave groups based on JONSWAP spectra with $\gamma$ values of $1,3.3$, and 6.6 (dotted, solid, and dashed lines, respectively). We also illustrate the effects of directional spreading, for following-sea (black and gray lines) and crossing-sea conditions (red lines). When normalized directional spreading does not have a strong effect on the shape of the amplitude distribution. When plotted on a logarithmic scale, it becomes clear that the subharmonic amplitude distributions can be approximated by the form $y=\exp (a x+b)$. This simple approximate relation is less 
accurate for very narrow-banded conditions (large $\gamma$ ). For broadbanded conditions $(\gamma \approx 3.3)$, this approximate relation can be used to estimate the ratio of subharmonic contribution to crest height filtered out to the total subharmonic contribution to crest height,

$$
\varepsilon_{\mathrm{hpf}}=\frac{\int_{f_{c} / f_{p}}^{\infty} e^{\left(a f / f_{p}+b\right)} d f}{\int_{0}^{\infty} e^{\left(a f / f_{p}+b\right)} d f}
$$

which simplifies to,

$$
\varepsilon_{\mathrm{hpf}}=e^{a f_{c} / f_{p}}
$$

where the gradient $a$ is derived from the data in Fig. D1a: $a \approx-2.0$. Figure D1b illustrates this process schematically.

\section{REFERENCES}

Allender, J., T. Audunson, S. F. Barstow, S. Bjerken, H. E. Krogstad, P. Steinbakke, L. Vartdal, and C. G. L. E. Borgman, 1989: The WADIC project: A comprehensive field evaluation of directional wave instrumentation. Ocean Eng., 16, 505-536, https://doi.org/10.1016/00298018(89)90050-4.

Bidlot, J., D. J. Holmes, P. A. Wittmann, R. Lalbeharry, and H. S. Chen, 2002: Intercomparison of the performance of operational ocean wave forecasting systems with buoy data. Wea. Forecasting, 17, 287-310, https://doi.org/10.1175/1520-0434(2002)017<0287: IOTPOO $>2.0 . \mathrm{CO} ; 2$.

Boccotti, P., 1981: On the highest waves in a stationary Gaussian process. Atti Accad. Ligure Sci. Lett., 38, 271-302.

_- 1983: Some new results on statistical properties of wind waves. Appl. Ocean Res., 5, 134-140, https://doi.org/10.1016/ 0141-1187(83)90067-6.

Casas-Prat, M., and L. H. Holthuijsen, 2010: Short-term statistics of waves observed in deep water. J. Geophys. Res., 115, C09024, https://doi.org/10.1029/2009JC005742.

Christou, M., and K. Ewans, 2011: Examining a comprehensive dataset containing thousands of freak wave events: Part 2-Analysis and findings. Structures, Safety and Reliability, Vol. 2, Proceedings of the ASME 2011 30th International Conference on Ocean, Offshore and Arctic Engineering, ASME, 827-837, https://doi.org/10.1115/OMAE2011-50169827-837.

_ J. Phys. Oceanogr., 44, 2317-2335, https://doi.org/10.1175/ JPO-D-13-0199.1.

Collins, C. O., III, B. Lund, T. Waseda, and H. C. Graber, 2014: On recording sea surface elevation with accelerometer buoys: lessons from ITOP (2010). Ocean Dyn., 64, 895-904, https:// doi.org/10.1007/s10236-014-0732-7.

Dalzell, J. F., 1999: A note on finite depth second-order wave-wave interactions. Appl. Ocean Res., 21, 105-111, https://doi.org/ 10.1016/S0141-1187(99)00008-5.

Datawell BV, 2019: Datawell Waverider Manual DWR4. http:// www.datawell.nl/Support/Documentation/Manuals.aspx.

de Vries, J. J., J. Waldron, and V. Cunningham, 2003: Field tests of the new Datawell DWR-G GPA wave buoy. Sea Technol., $\mathbf{4 4}$ $50-55$.
Dysthe, K. B., H. E. Krogstad, and P. Müller, 2008: Oceanic rogue waves. Annu. Rev. Fluid Mech., 40, 287-310, https://doi.org/ 10.1146/annurev.fluid.40.111406.102203.

Fedele, F., and M. A. Tayfun, 2009: On nonlinear wave groups and crest statistics. J. Fluid Mech., 620, 221-239, https://doi.org/ 10.1017/S0022112008004424.

— and F. Arena, 2010: Long-term statistics and extreme waves of sea storms. J. Phys. Oceanogr., 40, 1106-1117, https:// doi.org/10.1175/2009JPO4335.1.

Forristall, G. Z., 2000: Wave crest distributions: Observations and second-order theory. J. Phys. Oceanogr., 30, 1931-1943, https://doi.org/10.1175/1520-0485(2000)030<1931:WCDOAS> 2.0.CO;2.

Hasselmann, D. E., M. Dunckel, and J. A. Ewing, 1980: Directional wave spectra observed during JONSWAP 1973. J. Phys. Oceanogr., 10, 1264-1280, https://doi.org/10.1175/1520-0485(1980) 010<1264:DWSODJ $>2.0 . C O ; 2$.

Hasselmann, K., and Coauthors, 1973: Measurements of windwave growth and swell decay during the Joint North Sea Wave Project (JONSWAP). Deutschen Hydrographischen Zeitschrift Ergänzungsheft 8-12, 95 pp.

Herbers, T. H. C., and T. T. Janssen, 2016: Lagrangian surface wave motion and Stokes drift fluctuations. J. Phys. Oceanogr., 46, 1009-1021, https://doi.org/10.1175/JPO-D-15-0129.1.

Janssen, P. A. E. M., B. Hansen, and J. Bidlot, 1997: Verification of the ECMWF wave forecasting system against buoy and altimeter data. Wea. Forecasting, 12, 763-784, https://doi.org/10.1175/ 1520-0434(1997)012<0763:VOTEWF > 2.0.CO;2.

Krogstad, H. E., and S. F. Barstow, 2000: A unified approach to extreme value analysis of ocean waves. Tenth Int. Offshore and Polar Engineering Conf., Seattle, WA, International Society of Offshore and Polar Engineers, ISOPE-I-00-230, https://www.onepetro.org/conference-paper/ISOPE-I-00-230.

Latheef, M., and C. Swan, 2013: A laboratory study of wave crest statistics and the role of directional spreading. Proc. Roy. Soc. London, 469A, 20120696, https://doi.org/10.1098/rspa.2012.0696.

Lenee-Bluhm, P., R. Paasch, and H. T. Özkan-Haller, 2011: Characterizing the wave energy resource of the US Pacific Northwest. Renew. Energy, 36, 2106-2119, https://doi.org/10.1016/ j.renene.2011.01.016.

Lindgren, G., 1970: Some properties of a normal process near a local maximum. Ann. Math. Stat., 41, 1870-1883, https://doi.org/ 10.1214/aoms/1177696688.

Longuet-Higgins, M. S., 1952: On the statistical distribution of the heights of sea waves. J. Mar. Res., 11, 245-266.

- , 1980: On the distribution of the heights of sea waves: Some effects of nonlinearity and finite band width. J. Geophys. Res., 85, 1519-1523, https://doi.org/10.1029/JC085iC03p01519.

_ 1986: Eulerian and Lagrangian aspects of surface waves. J. Fluid Mech., 173, 683-707, https://doi.org/10.1017/S0022112086001325.

Marthinsen, T., and S. R. Winterstein, 1992: On the skewness of random surface waves. Second Int. Offshore and Polar Engineering Conf., San Francisco, CA, International Society of Offshore and Polar Engineers, ISOPE-I-92-279, https:// www.onepetro.org/conference-paper/ISOPE-I-92-279.

McAllister, M. L., and T. S. van den Bremer, 2019: Lagrangian measurement of steep directionally spread ocean waves: Second-order motion of a wave-following measurement buoy. J. Phys. Oceanogr., 49, 3087-3108, https://doi.org/10.1175/JPO-D-19-0170.1.

, T. A. A. Adcock, T. S. van den Bremer, and P. H. Taylor, 2019: A note on the second-order contribution to extreme waves generated during hurricanes. J. Offshore Mech. Arct. Eng., 141, 041102, https://doi.org/10.1115/1.4042540. 
Miles, M. D., and E. R. Funke, 1989: A comparison of methods for synthesis of directional seas. J. Offshore Mech. Arct. Eng., 111, 43-48, https://doi.org/10.1115/1.3257137.

Mork, G., S. Barstow, A. Kabuth, and M. T. Pontes, 2010: Assessing the global wave energy potential. Proceedings of the ASME 201029 th International Conference on Ocean, Offshore and Arctic Engineering, Vol. 3, ASME, 447-454, https:// doi.org/10.1115/OMAE2010-20473

Onorato, M., and Coauthors, 2009: Statistical properties of mechanically generated surface gravity waves: A laboratory experiment in a three-dimensional wave basin. J. Fluid Mech., 627, 235-257, https://doi.org/10.1017/S002211200900603X.

Pontes, M. T., 1998: Assessing the European wave energy resource. J. Offshore Mech. Arct. Eng., 120, 226-231, https://doi.org/ 10.1115/1.2829544.

Prevosto, M., H. E. Krogstad, and R. Agnès, 2000: Probability distributions for maximum wave and crest heights. Coast. Eng., 40, 329-360, https://doi.org/10.1016/S0378-3839(00)00017-X.

Raghukumar, K., G. Chang, F. Spada, C. Jones, T. Janssen, and A. Gans, 2019: Performance characteristics of "Spotter," a newly developed real-time wave measurement buoy. J. Atmos. Oceanic Technol., 36, 1127-1141, https://doi.org/10.1175/JTECHD-18-0151.1.

Santo, H., P. H. Taylor, R. Eatock Taylor, and Y. S. Choo, 2013: Average properties of the largest waves in Hurricane Camille. J. Offshore Mech. Arct. Eng., 135, 011602, https://doi.org/ 10.1115/1.4006930.

Seymour, R. J., and D. Castel, 1998: Systematic underestimation of maximum crest heights in deep water using surface-following buoys. 17th Int. Conf. on Offshore Mechanics and Arctic Engineering, Lisbon, Portugal, ASME, OMAE98-1466.
Socquet-Juglard, H., K. Dysthe, and K. Trulsen, 2005: Probability distributions of surface gravity waves during spectral changes. J. Fluid Mech., 542, 195-216, https://doi.org/10.1017/S0022112005006312.

Srokosz, M. A., and M. S. Longuet-Higgins, 1986: On the skewness of sea-surface elevation. J. Fluid Mech., 164, 487-497, https:// doi.org/10.1017/S0022112086002653.

Tayfun, M. A., 1980: Narrow-band nonlinear sea waves. J. Geophys. Res., 85, 1548-1552, https://doi.org/10.1029/JC085iC03p01548.

—, and F. Fedele, 2007: Wave-height distributions and nonlinear effects. Ocean Eng., 34, 1631-1649, https://doi.org/10.1016/ j.oceaneng.2006.11.006.

Tromans, P. S., A. R. Anaturk, and P. Hagemjier, 1991: A new model for the kinematics of large ocean waves-application as a design wave. First Int. Offshore and Polar Engineering Conf., Edinburgh, United Kingdom, International Society of Offshore and Polar Engineers, 8 pp., https://www.onepetro.org/conferencepaper/ISOPE-I-91-154.

Tucker, M. J., P. G. Challenor, and D. J. T. Carter, 1984: Numerical simulation of a random sea: A common error and its effect upon wave group statistics. Appl. Ocean Res., 6, 118-122, https://doi.org/10.1016/0141-1187(84)90050-6.

van Essen, S., K. Ewans, and J. McConichie, 2018: Wave buoy performance in short and long waves, evaluated using tests on a hexapod. 37th Int. Conf. on Ocean, Offshore and Arctic Engineering, Madrid, Spain, OMAE2018-77092, https://doi.org/ 10.1115/OMAE2018-77092.

Whittaker, C. N., A. C. Raby, C. J. Fitzgerald, and P. H. Taylor, 2016: The average shape of large waves in the coastal zone. Coast. Eng., 114, 253-264, https://doi.org/10.1016/j.coastaleng.2016.04.009.

Young, I. R., 2006: Directional spectra of hurricane wind waves. J. Geophys. Res., 111, C08020, https://doi.org/10.1029/2006JC003540. 